\title{
Important role of screening the electron-hole exchange interaction for the optical properties of molecules near metal surfaces
}

\author{
Deilmann, Thorsten; Thygesen, Kristian Sommer
}

Published in:

Physical Review B

Link to article, DOI:

10.1103/PhysRevB.99.045133

Publication date:

2019

Document Version

Publisher's PDF, also known as Version of record

Link back to DTU Orbit

Citation $(A P A)$ :

Deilmann, T., \& Thygesen, K. S. (2019). Important role of screening the electron-hole exchange interaction for the optical properties of molecules near metal surfaces. Physical Review B, 99(4), [045133].

https://doi.org/10.1103/PhysRevB.99.045133

\section{General rights}

Copyright and moral rights for the publications made accessible in the public portal are retained by the authors and/or other copyright owners and it is a condition of accessing publications that users recognise and abide by the legal requirements associated with these rights.

- Users may download and print one copy of any publication from the public portal for the purpose of private study or research.

- You may not further distribute the material or use it for any profit-making activity or commercial gain

- You may freely distribute the URL identifying the publication in the public portal 


\title{
Important role of screening the electron-hole exchange interaction for the optical properties of molecules near metal surfaces
}

\author{
Thorsten Deilmann ${ }^{1, *}$ and Kristian Sommer Thygesen ${ }^{1,2}$ \\ ${ }^{1}$ CAMD, Department of Physics, Technical University of Denmark, DK-2800 Kongens Lyngby, Denmark \\ ${ }^{2}$ Center for Nanostructured Graphene (CNG), Technical University of Denmark, DK-2800 Kongens Lyngby, Denmark
}

(Received 9 November 2018; revised manuscript received 21 December 2018; published 18 January 2019)

\begin{abstract}
Optical experiments on nanostructures such as molecules, one- or two-dimensional materials, are often performed with the nanostructures in close proximity of a substrate or some other polarizable media. In this case, the Bethe-Salpeter equation (BSE) can be used to calculate the optical excitations of the nanostructure by including the effect of the substrate via the screened electron-hole interaction. Here we show, that in such an approach, where the states of the substrate are not explicitly included in the BSE Hamiltonian but only enter through the screened Coulomb interaction, it is important also to screen the electron-hole exchange interaction. For the case of molecules like benzene physisorbed on the metallic $\mathrm{Au}(111)$ surface, the screening of the exchange interaction by the substrate redshifts the lowest optical transition by up to $300 \mathrm{meV}$. Furthermore, the screening of the exchange is essential in order to obtain the correct ordering of the size of quasiparticle and optical energy gap.
\end{abstract}

DOI: 10.1103/PhysRevB.99.045133

\section{INTRODUCTION}

Over the past decade, the fields of nano-optics and nanooptoelectronics have developed rapidly [1-5]. In these fields, the study of optical transitions in nanoscale structures, such as molecules, one-dimensional (1D) or 2D materials, plays a fundamentally important role. It is well known, that the optical properties of a nanostructure can be strongly influenced by the environment, and in fact this kind of interaction may even be exploited to modify the properties of the nanostructure [6-8]. Specifically, the light-matter interaction achieved in molecule-metal structures constitute the basis for applications such as molecular sensors $[9,10]$ and surface enhanced Raman spectroscopy [11-13]. Moreover, such structures form the basis of recent efforts to achieve strong coupling between molecular excitations and surface plasmons [14,15].

An accurate theoretical description is not possible using the density functional theory (DFT) which cannot account for the dielectric screening of the surroundings. In fact, it is well known that even the single-particle energy levels (also known as the quasiparticle energy levels) of molecules on a polarizable substrate, are strongly renormalized due to image charge screening effects [16-18]. This effect leads to an upshift of the occupied molecular levels and downshift of the unoccupied levels, and thus it effectively closes the gap. While the effect can be very significant, i.e., on the order of several electron volts, and is essential to include in order to reproduce transport experiments on molecular junctions [19], it is completely missed by density functional theory with common approximations to the exchange-correlation functional. In this work we take a step beyond the description of the quasiparticle levels, and consider the effect of substrate screening on the optical excitations. The state-of-the art technique to describe

*thorsten.deilmann@wwu.de the optical properties from first principles is the $G W / \mathrm{BSE}$ approach $[20,21]$. For example, this approach has been used successfully to describe the redshift of both the quasiparticle gap and optical excitations [16,22,23].

In the $G W / \mathrm{BSE}$ formulation the exchange interaction enters in its bare form in contrast to the direct electron-hole interaction which must be screened. The solution of the BSE for the combined system of a molecule and a metallic substrate is computationally demanding due to the large number of involved states. As a result, an attractive solution is to construct the BSE Hamiltonian in a restricted part of the Hilbert space representing only the adsorbate and include the states of the substrate indirectly via its effect on the screened Coulomb interaction. In Ref. [24], Spataru used such an approach to investigate the influence of a metallic substrate on the optical excitations in a carbon nanotube. In this case, rather minor effects of the substrate on the optical gap of a few tens of meV were obtained. Here we show that effects due to substrate screening can be much larger for molecular adsorbates. In particular, we show the importance of screening the electron-hole exchange interaction, which has often been neglected or overlooked in the literature. In fact, an insufficient screening of the exchange term can result in an optical band gap exceeding the quasiparticle gap.

The paper is organized as follows. In Sec. II we derive the response function of a weakly physisorbed nanostructure if the substrate response is only included by its dielectric response. After the description of its implementation in Sec. III we finally apply this to the test system of benzene on $\mathrm{Au}(111)$ in Sec. IV.

\section{THEORY}

The Bethe-Salpeter equation (BSE) is typically written as [20]

$$
\chi=\chi^{0}+\chi^{0} K \chi,
$$


where $\chi\left(\chi^{0}\right)$ is the (noninteracting) response function. In the $G W$ approximation the interaction kernel can be written as the screened direct interaction and the bare exchange interaction $K=v-W$. The screening of the nanostructure and the surrounding can be easily included in $W[22,25]$, namely this term can reliably describe both situations, a molecule in vacuum and on a substrate. Thus we will only focus on the bare exchange interaction $K=v$ in the following derivations which corresponds to the RPA. We can formally solve Eq. (1) as

$$
\chi=\left(1-\chi^{0} v\right)^{-1} \chi^{0} .
$$

By inserting this back in Eq. (1) we find

$$
\chi=\chi^{0}+\chi^{0} v\left(1-\chi^{0} v\right)^{-1} \chi^{0} .
$$

Typically the BSE is solved including a few valence and conduction bands as "active space" [20,21], i.e., considering all possible linear combination of the transitions between these states. In many systems this is already computationally demanding. As described above, the substrate can be implicitly taken into account in the BSE via modifying the screened interaction $W$ that determines the direct electronhole interaction. On the other hand, the electronic states of the substrate are not explicitly included in the BSE and thus the bare exchange would not be modified.

In the following we will divide the response function in two subspaces, e.g., the nanostructure/molecular orbitals $(m)$ and the surface/rest $(r)$ and write it as a block matrix

$$
\chi \Rightarrow\left(\begin{array}{cc}
\chi_{m, m} & \chi_{m, r} \\
\chi_{r, m} & \chi_{r, r}
\end{array}\right) .
$$

This approach is similar to the derivations of partially screened Hubbard $U$ parameters in which the screening of $d$ orbitals is separated [26,27], as well as the procedure used in Ref. [24]. We also refer to a more general discussion on this topic by Benedict [28]. By the projection operator

$$
P_{m}=\left(\begin{array}{ll}
1 & 0 \\
0 & 0
\end{array}\right)
$$

we are able to extract the subspace $m$, i.e., its BSE is set up as

$$
\begin{aligned}
P_{m} \chi P_{m} & =P_{m} \chi^{0} P_{m}+P_{m} \chi^{0} v \chi P_{m} \\
& \stackrel{(2)}{=} P_{m} \chi^{0} P_{m}+P_{m} \chi^{0} v\left(1-\chi^{0} v\right)^{-1} \chi^{0} P_{m},
\end{aligned}
$$

which can be written as

$$
\begin{aligned}
\left(\begin{array}{cc}
\chi_{m, m} & 0 \\
0 & 0
\end{array}\right)= & \left(\begin{array}{cc}
\chi_{m, m}^{0} & 0 \\
0 & 0
\end{array}\right)+\left(\begin{array}{cc}
\chi_{m, m}^{0} & \chi_{m, r}^{0} \\
0 & 0
\end{array}\right) \\
& \times v\left[\left(\begin{array}{ll}
1 & 0 \\
0 & 1
\end{array}\right)-\left(\begin{array}{cc}
\chi_{m, m}^{0} & \chi_{m, r}^{0} \\
\chi_{r, m}^{0} & \chi_{r, r}^{0}
\end{array}\right) v\right]^{-1} \\
& \times\left(\begin{array}{cc}
\chi_{m, m}^{0} & 0 \\
\chi_{r, m}^{0} & 0
\end{array}\right) .
\end{aligned}
$$

Here $\chi^{0} / \chi$ are four point functions which are set up in an electron-hole pair basis. The matrix notation $\chi_{i, j}$ denotes that the holes/electrons are restricted to the subspace $i / j$ where $i, j \in m, r$. The fact that $\chi_{0}$ takes a diagonal form in this block representation follows from the assumption of vanishing overlap of wave functions belonging to the molecule and substrate, respectively. The assumption of vanishing overlap between the wave functions of the two subsystems should be a good approximation, e.g., for weakly physisorbed molecules, and represents a simplifying approximation for more strongly bound molecules. After expanding Eq. (3) [see appendix Eq. (A2)] we set $\chi_{m, r}^{0}=0$ and $\chi_{r, m}^{0}=0$. In the following we are only interested in the first block of the matrix which describes the neutral excitations of the molecule in the presence of the substrate

$$
\chi_{m, m}=\chi_{m, m}^{0}+\chi_{m, m}^{0} v\left[1-\chi_{m, m}^{0} v-\chi_{r, r}^{0} v\right]^{-1} \chi_{m, m}^{0} .
$$

In contrast, if we had disregarded the orbitals of the substrate from the beginning, we would have incorrectly obtained

$$
\tilde{\chi}_{m, m}=\chi_{m, m}^{0}+\chi_{m, m}^{0} v\left[1-\chi_{m, m}^{0} v\right]^{-1} \chi_{m, m}^{0} .
$$

To elaborate on the differences we transform Eq. (4) by extending with $1 \equiv\left(1-\chi_{r r}^{0} v\right)^{-1}\left(1-\chi_{r r}^{0} v\right)$,

$$
\begin{aligned}
\chi_{m, m}= & \chi_{m, m}^{0}+\chi_{m, m}^{0} v\left(1-\chi_{r r}^{0} v\right)^{-1} \\
& \times\left(1-\chi_{r r}^{0} v\right)\left[\left(1-\chi_{r, r}^{0} v\right)-\chi_{m, m}^{0} v\right]^{-1} \chi_{m, m}^{0} \\
= & \chi_{m, m}^{0}+\chi_{m, m}^{0} v\left(1-\chi_{r r}^{0} v\right)^{-1} \\
& \times\left[1-\chi_{m, m}^{0} v\left(1-\chi_{r r}^{0} v\right)^{-1}\right]^{-1} \chi_{m, m}^{0} \\
\equiv & \chi_{m, m}^{0}+\chi_{m, m}^{0} v_{\mathrm{scr}}\left[1-\chi_{m, m}^{0} v_{\mathrm{scr}}\right]^{-1} \chi_{m, m}^{0} .
\end{aligned}
$$

Comparing $\chi_{m, m}$ with $\tilde{\chi}_{m, m}$ in Eq. (5) we find that additional screening is introduced by the surface.In Fig. 1 this is shown in terms of Feynman diagrams. We find that $\tilde{\chi}_{m, m}$ in Eq. (5) becomes $\chi_{m, m}$ if the bare Coulomb is replaced with a Coulomb interaction $v_{\text {scr }}=v\left(1-\chi_{r r}^{0} v\right)^{-1}$ that is screened by part $r$, i.e., by the states excluded from the BSE. We note that $\chi_{r r}^{0}$ does not include excitonic effects and reduces to a two-point function which is calculated within the RPA [24].

Again we underline that this result implies that we are describing two separate parts between which the "overlap" $\chi_{m, r}^{0}$ can be neglected. However, we note that we expect further (but smaller) modifications for the exchange if bands of the same subsystem are not included, e.g., if only the highest occupied molecular orbital (HOMO) and lowest unoccupied molecular orbital (LUMO) of the molecule are used.

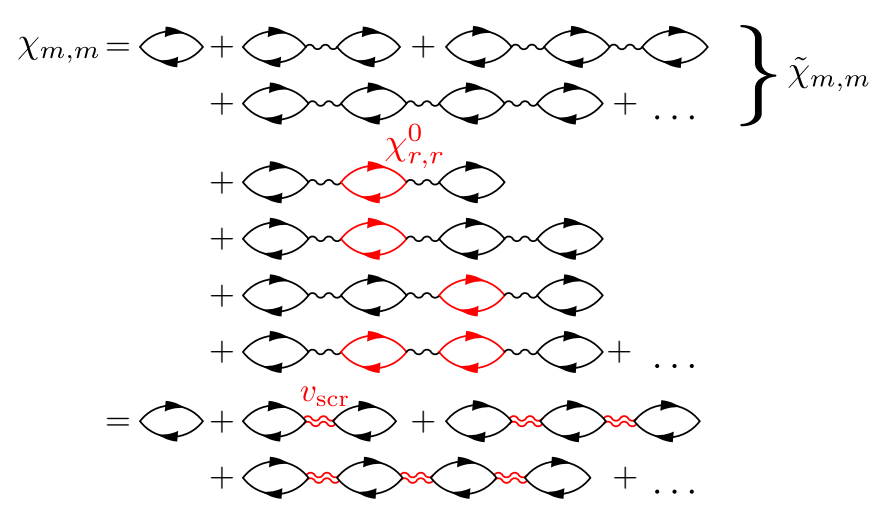

FIG. 1. Equation (6) in terms of Feynman diagrams. The noninteraction response functions corresponding to the surface $\chi_{r, r}^{0}$ are indicated in red while those of the molecule $\chi_{m, m}^{0}$ are black. 


\section{IMPLEMENTATION}

After we have rewritten the BSE for the exchange part using $v_{\text {scr }}$ [Eq. (6)] we add the direct interaction again and transform it to an eigenvalue problem [20,21] with matrix elements (in the Tamm-Dancoff approximation [29]),

$$
\left\langle\mathbf{v} \mathbf{c}\left|\hat{H}^{(e h)}\right| \mathbf{v}^{\prime} \mathbf{c}^{\prime}\right\rangle=\left(\epsilon_{\mathbf{c}}-\epsilon_{\mathbf{v}}\right) \delta_{\mathbf{c c}^{\prime}} \delta_{\mathbf{v v}^{\prime}}-\left(W_{\mathbf{v}^{\prime} \mathbf{c}, \mathbf{v} \mathbf{c}^{\prime}}-V_{\mathbf{v}^{\prime} \mathbf{c}, \mathbf{c}^{\prime} \mathbf{v}}^{\mathrm{src}}\right) \text {. }
$$

The matrix elements of the direct interaction $W\left(\mathbf{r}, \mathbf{r}^{\prime}\right)=$ $\int \varepsilon^{-1}\left(\mathbf{r}, \mathbf{r}^{\prime \prime}\right) V\left(\mathbf{r}^{\prime \prime}, \mathbf{r}^{\prime}\right) d^{3} r^{\prime \prime}$ are screened by the dielectric function of the complete system, i.e., by the screening of the nanostructure as well as the dielectric substrate including $\chi_{r r}^{0}$. In addition the bare exchange has been replaced by the "substrate-screened" exchange

$$
V\left(\mathbf{r}, \mathbf{r}^{\prime}\right) \rightarrow V^{\mathrm{scr}}\left(\mathbf{r}, \mathbf{r}^{\prime}\right)=\int \tilde{\varepsilon}^{-1}\left(\mathbf{r}, \mathbf{r}^{\prime \prime}\right) V\left(\mathbf{r}^{\prime \prime}, \mathbf{r}^{\prime}\right) d^{3} r^{\prime \prime},
$$

i.e., $\tilde{\varepsilon}$ is the dielectric screening of the substrate $\left(\chi_{r r}^{0}\right)$ only.

In our DFT we employ norm-conserving pseudopotentials in the Kleinman-Bylander form [30,31] and a basis of localized atom-centered Gaussian orbitals of $s, p, d$, and $s^{*}$ symmetry [32]. For the benzene molecule we employ all 15 occupied and 15 unoccupied orbitals consistent in all DFT, $G d W$, and BSE calculations. The screening of benzene is calculated employing the RPA and mapped onto atomresolved model functions; the surface screening is described by metallic model functions. For a detailed discussion of the many-body approach and the dielectric function we refer to Refs. [23,33].

\section{BENZENE ON GOLD(111)}

After the derivation and the implementation of the BSE for a nanostructure above a substrate in Secs. II and III, we will employ this approach for a benzene molecule above the metallic Au(111) surface.

Benzene [Figs. 2(a) and 2(b)] is the most simple aromatic molecule consisting of a ring of six carbon atoms with one hydrogen atom attached to each of them. The molecule is planar and its bond lengths are given by $d_{\mathrm{CC}}=1.41 \AA$ and $d_{\mathrm{CH}}=1.11 \AA$. Employing the $G d W(\mathrm{LDA})$ approximation [25] in vacuum we find a HOMO-LUMO gap of $10.68 \mathrm{eV}$ in very good agreement with our $G W$ result of $10.66 \mathrm{eV}$ and the literature $(10.51 \mathrm{eV})$ [16].

In the next step we examine the benzene molecule in the vicinity of an $\mathrm{Au}(111)$ surface. The system is employed in a $6 \times 6$ supercell with six layers of $\mathrm{Au}$; the surface is included by its dielectric polarizability [25]. For different distances $d$ the resulting quasiparticle HOMO-LUMO gap is shown in Fig. 2(c) (black dots). Its dependency has been fitted by $10.68 \mathrm{eV}-7.93 \mathrm{e} V \AA /(d-0.21 \AA)$. We note that for distances $d<3 \AA$ the overlap is no longer negligible and the results should be interpreted with care. The optical HOMOLUMO gap as result of the BSE is compared using the bare exchange (blue) and the surface-screened exchange (red). For large $d$ both excitation energies are almost identical, because the screening due to the surface vanishes. Two important effects can be observed at smaller distances: (i) The exciton energy employing the bare exchange is nearly constant (slightly blueshifted) if the molecule moves closer to the surface. In
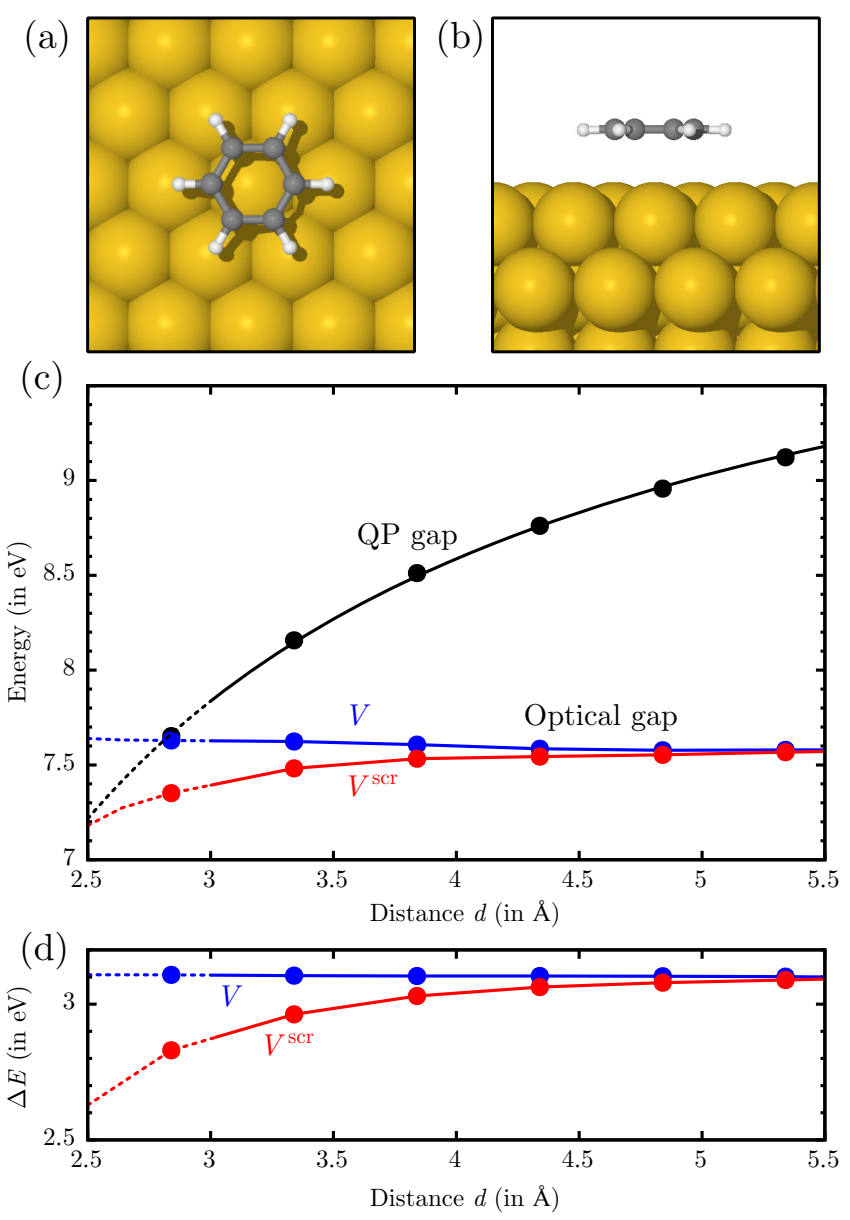

FIG. 2. (a),(b) Top and side view of benzene on the $\mathrm{Au}(111)$ surface. (c) Quasiparticle (QP) HOMO-LUMO gap (black) and optical HOMO-LUMO gap employing the bare (blue) and substratescreened (red) exchange interaction. For distances smaller than $3 \AA$ the overlap of benzene and surface is no longer negligible. (d) Singlet-triplet splitting $\Delta E$ of the corresponding $E_{1 \mathrm{u}}$ state.

contrast to this $V^{\text {scr }}$ reduces the energy of the exciton, which is physically expected [7]. (ii) Consequently, employing $V$ leads to an electronic gap below the optical gap at a distance smaller than $2.8 \AA$. In the picture of excitons as bound electron-hole states this behavior is counterintuitive. Using the surfacescreened exchanged $V^{\text {scr }}$ the excitation energy is found below the QP gap with a decreased exciton binding energy. A further consequence of the surface-screened exchange $V^{\text {scr }}$ is the renormalization of the singlet-triplet splitting [see Fig. 2(d) for the $E_{1 \mathrm{u}}$ state]. We find a noticeable reduction of $0.22 \mathrm{eV}$ for a distance of $3 \AA$ employing $V^{\text {scr }}$ while the splitting is nearly constant excluding the surface-screened exchange. We note in passing that a replacement of $V^{\mathrm{scr}}$ with a fully screened $W$ would lead to much stronger effects, i.e., the splitting $\Delta E$ would reduce to $0.8 \mathrm{eV}$ nearly independent of the distance $d$.

We note that the energy of the QP gap follows a $1 / d$ behavior due to the image charge effect. Therefore, the calculation (or measurement) of the vacuum band gap (i.e., HOMO-LUMO gap) requires a huge distance ( $\gg 5 \AA)$ to further polarizable dielectric media/atoms. On the other hand, the modification of the excitation energy due to the screening 
of the exchange $V^{\text {scr }}$ is weaker and we find that it only becomes significant if the adsorbent is closer than $\sim 4.5 \AA$. The exchange matrix elements $V_{\mathbf{v}^{\prime} \mathbf{c}, \mathbf{c}^{\prime} \mathbf{v}}^{\mathrm{sr}}$ in Eq. (7) mediate between an excitation $\mathbf{v} \rightarrow \mathbf{c}$ (at $r$ ) and $\mathbf{v}^{\prime} \rightarrow \mathbf{c}^{\prime}$ (at $r^{\prime}$ ). As only dipole (and higher) moments occur for the exchange, its interaction decays more rapidly as compared to the direct term.

Benzene on a metallic $\mathrm{Al}(111)$ surface has been investigated previously [22]. In qualitative agreement to our unscreened results, it was found that the optical gap can drop the quasiparticle gap. Due to different substrates and the numerical different treatment it was already observed for a distance of $d=4.5 \AA$ A. Here we conclude that the incorrect ordering of the optical and QP gaps can be explained by uncorrected usage of the exchange interaction in the BSE. In comparison to the case of carbon nanotubes [24] the renormalization of the optical band gap of benzene is distinctly stronger. This emphasizes the importance of a balanced modification of the direct and exchange interaction due to the surface screening.

\section{CONCLUSION}

In summary, we have implemented a correction for the exchange interaction employed in the BSE. The correction should be employed in calculations of nanostructures in the vicinity of a surface/polarizable substrate whenever the states of the substrate are only included implicitly in the BSE via the dielectric screening. In this case the dielectric screening has to be included in the exchange. Thus the largest effects can be expected near metallic surfaces in systems with large exchange contributions. For benzene on $\mathrm{Au}(111)$ we find that this screening corrects the unphysical behavior of the quasiparticle HOMO-LUMO gap dropping below the optical HOMO-LUMO gap as observed in previous studies [22]. In general the substrate screening reduces the exchange and therefore redshifts the excitons.

\section{ACKNOWLEDGMENTS}

T.D. acknowledges the financial support from the Villum foundation. The Center for Nanostructured Graphene is sponsored by the Danish National Research Foundation, Project DNRF103. This project has received funding from the European Research Council (ERC) under the European Union's Horizon 2020 research and innovation programme (Grant Agreement No. 773122, LIMA).

\section{APPENDIX}

For a block matrix it is well known that it can be inverted by

$$
\left(\begin{array}{ll}
A & B \\
C & D
\end{array}\right)^{-1}=\left(\begin{array}{cc}
S^{-1} & -S^{-1} B D^{-1} \\
-D^{-1} C S^{-1} & D^{-1}+D^{-1} C S^{-1} B D^{-1}
\end{array}\right)
$$

with the Schur complement $S=A-B D^{-1} C$. For the special form of (we assume both subsystems to have the same number of bands)

$$
A=1-X, \quad B=-X, \quad C=-Y, \quad \text { and } \quad D=1-Y
$$

$S$ simplifies to $(1-Y)(1-X-Y)^{-1}$. Thus the inversion in Eq. (A1) can be rewritten as

$$
\left(\begin{array}{cc}
1-X & -X \\
-Y & 1-Y
\end{array}\right)^{-1}=\left(\begin{array}{cc}
(1-Y)(1-X-Y)^{-1} & (1-Y)(1-X-Y)^{-1} X(1-Y)^{-1} \\
Y(1-X-Y)^{-1} & (1-Y)^{-1}+Y(1-X-Y)^{-1} X(1-Y)^{-1}
\end{array}\right)
$$

[1] M. Eremtchenko, J. A. Schaefer, and F. S. Tautz, Understanding and tuning the epitaxy of large aromatic adsorbates by molecular design, Nature (London) 425, 602 (2003).

[2] T. Esat, B. Lechtenberg, T. Deilmann, C. Wagner, P. Krüger, R. Temirov, M. Rohlfing, F. B. Anders, and F. S. Tautz, A chemically driven quantum phase transition in a two-molecule Kondo system, Nat. Phys. 12, 867 (2016).

[3] J. Cai, P. Ruffieux, R. Jaafar, M. Bieri, T. Braun, S. Blankenburg, M. Muoth, A. P. Seitsonen, M. Saleh, X. Feng, K. Müllen, and R. Fasel, Atomically precise bottom-up fabrication of graphene nanoribbons, Nature (London) 466, 470 (2010).

[4] T. Low, A. Chaves, J. D. Caldwell, A. Kumar, N. X. Fang, P. Avouris, T. F. Heinz, F. Guinea, L. Martin-Moreno, and F. Koppens, Polaritons in layered two-dimensional materials, Nat. Mater. 16, 182 (2016).

[5] K. S. Thygesen, Calculating excitons, plasmons, and quasiparticles in 2D materials and van der Waals heterostructures, 2D Mater. 4, 022004 (2017).

[6] A. Raja, A. Chaves, J. Yu, G. Arefe, H. M. Hill, A. F. Rigosi, T. C. Berkelbach, P. Nagler, C. Schüller, T. Korn,
C. Nuckolls, J. Hone, L. E. Brus, T. F. Heinz, D. R. Reichman, and A. Chernikov, Coulomb engineering of the bandgap and excitons in two-dimensional materials, Nat. Commun. 8, 15251 (2017).

[7] M. Drüppel, T. Deilmann, P. Krüger, and M. Rohlfing, Diversity of trion states and substrate effects in the optical properties of an $\mathrm{MoS}_{2}$ monolayer, Nat. Commun. 8, 2117 (2017).

[8] K. T. Winther and K. S. Thygesen, Band structure engineering in van der Waals heterostructures via dielectric screening: The G $\Delta \mathrm{W}$ method, 2D Mater. 4, 025059 (2017).

[9] K. Kneipp, Y. Wang, H. Kneipp, L. T. Perelman, I. Itzkan, R. R. Dasari, and M. S. Feld, Single Molecule Detection Using Surface-Enhanced Raman Scattering (SERS), Phys. Rev. Lett. 78, 1667 (1997).

[10] M. Feierabend, G. Berghäuser, A. Knorr, and E. Malic, Proposal for dark exciton based chemical sensors, Nat. Commun. 8, 14776 (2017).

[11] P. C. Lee and D. Meisel, Adsorption and surface-enhanced raman of dyes on silver and gold sols, J. Phys. Chem. 86, 3391 (1982). 
[12] S. Nie and S. R. Emory, Probing single molecules and single nanoparticles by surface-enhanced raman scattering, Science 275, 1102 (1997).

[13] A. Campion and P. Kambhampati, Surface-enhanced raman scattering, Chem. Soc. Rev. 27, 241 (1998).

[14] A. J. Haes, S. Zou, J. Zhao, G. C. Schatz, and R. P. Van Duyne, Localized surface plasmon resonance spectroscopy near molecular resonances, J. Am. Chem. Soc. 128, 10905 (2006).

[15] P. Zijlstra, P. M. R. Paulo, and M. Orrit, Optical detection of single non-absorbing molecules using the surface plasmon resonance of a gold nanorod, Nat. Nanotechnol. 7, 379 (2012).

[16] J. B. Neaton, M. S. Hybertsen, and S. G. Louie, Renormalization of Molecular Electronic Levels at Metal-Molecule Interfaces, Phys. Rev. Lett. 97, 216405 (2006).

[17] J. M. Garcia-Lastra, C. Rostgaard, A. Rubio, and K. S. Thygesen, Polarization-induced renormalization of molecular levels at metallic and semiconducting surfaces, Phys. Rev. B 80, 245427 (2009).

[18] K. Kaasbjerg and K. Flensberg, Strong polarization-induced reduction of addition energies in single-molecule nanojunctions, Nano Lett. 8, 3809 (2008).

[19] M. Strange, C. Rostgaard, H. Häkkinen, and K. S. Thygesen, Self-consistent gw calculations of electronic transport in thioland amine-linked molecular junctions, Phys. Rev. B 83, 115108 (2011).

[20] G. Onida, L. Reining, and A. Rubio, Electronic excitations: Density-functional versus many-body Green's-function approaches, Rev. Mod. Phys. 74, 601 (2002).

[21] M. Rohlfing and S. G. Louie, Electron-hole excitations and optical spectra from first principles, Phys. Rev. B 62, 4927 (2000).

[22] J. M. Garcia-Lastra and K. S. Thygesen, Renormalization of Optical Excitations in Molecules near a Metal Surface, Phys. Rev. Lett. 106, 187402 (2011).
[23] T. Esat, T. Deilmann, B. Lechtenberg, C. Wagner, P. Krüger, R. Temirov, F. B. Anders, M. Rohlfing, and F. S. Tautz, Transfering spin into an extended $\pi$ orbital of a large molecule, Phys. Rev. B 91, 144415 (2015).

[24] C. D. Spataru, Electronic and optical gap renormalization in carbon nanotubes near a metallic surface, Phys. Rev. B 88, 125412 (2013).

[25] M. Rohlfing, Electronic excitations from a perturbative LDA+GdW approach, Phys. Rev. B 82, 205127 (2010).

[26] F. Aryasetiawan, M. Imada, A. Georges, G. Kotliar, S. Biermann, and A. I. Lichtenstein, Frequency-dependent local interactions and low-energy effective models from electronic structure calculations, Phys. Rev. B 70, 195104 (2004).

[27] F. Aryasetiawan, K. Karlsson, O. Jepsen, and U. Schönberger, Calculations of hubbardufrom first-principles, Phys. Rev. B 74, 125106 (2006).

[28] L. X. Benedict, Screening in the exchange term of the electronhole interaction of the bethe-salpeter equation, Phys. Rev. B 66, 193105 (2002).

[29] S. Hirata and M. Head-Gordon, Time-dependent density functional theory within the Tamm-Dancoff approximation, Chem. Phys. Lett. 314, 291 (1999).

[30] D. R. Hamann, Generalized norm-conserving pseudopotentials, Phys. Rev. B 40, 2980 (1989).

[31] L. Kleinman and D. M. Bylander, Efficacious Form for Model Pseudopotentials, Phys. Rev. Lett. 48, 1425 (1982).

[32] T. Deilmann, P. Krüger, M. Rohlfing, and D. Wegner, Adsorption and STM imaging of tetracyanoethylene on $\mathrm{Ag}(001)$ : An ab initio study, Phys. Rev. B 89, 045405 (2014).

[33] M. Drüppel, T. Deilmann, J. Noky, P. Marauhn, P. Krüger, and M. Rohlfing, Electronic excitations in transition metal dichalcogenide monolayers from an LDA $+G d W$ approach, Phys. Rev. B 98, 155433 (2018). 